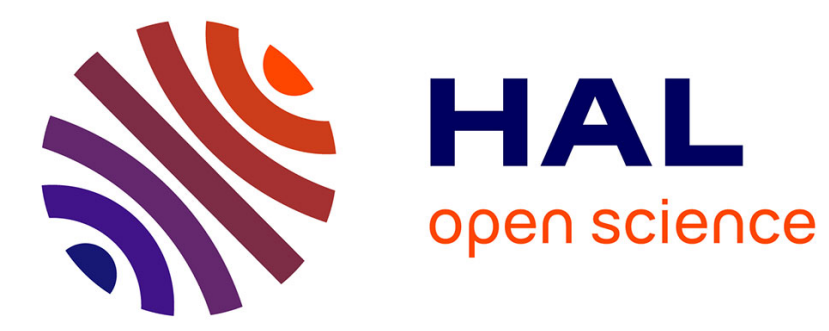

\title{
Study differentiating fish oocyte developmental stages using bioimpedance spectroscopy
}

Eloïse Detrez, Vincent Kerzérho, Mohamed Moez Belhaj, Alain Vergnet, Hugues de Verdal, Tristan Rouyer, Sylvain Bonhommeau, Achraf Lamlih, Mohan Julien, Fathi Ben Ali, et al.

\section{To cite this version:}

Eloïse Detrez, Vincent Kerzérho, Mohamed Moez Belhaj, Alain Vergnet, Hugues de Verdal, et al.. Study differentiating fish oocyte developmental stages using bioimpedance spectroscopy. Aquaculture, 2022, 547, pp.\#737396. 10.1016/j.aquaculture.2021.737396 . lirmm-03334895

\section{HAL Id: lirmm-03334895 https://hal-lirmm.ccsd.cnrs.fr/lirmm-03334895}

Submitted on 5 Sep 2021

HAL is a multi-disciplinary open access archive for the deposit and dissemination of scientific research documents, whether they are published or not. The documents may come from teaching and research institutions in France or abroad, or from public or private research centers.
L'archive ouverte pluridisciplinaire HAL, est destinée au dépôt et à la diffusion de documents scientifiques de niveau recherche, publiés ou non, émanant des établissements d'enseignement et de recherche français ou étrangers, des laboratoires publics ou privés. 


\title{
Study differentiating fish oocyte developmental stages using bioimpedance spectroscopy
}

\author{
Eloïse Detrez ${ }^{\mathrm{a}, \mathrm{b}}$, Vincent Kerzérho ${ }^{\mathrm{a}, *}$, Mohamed-Moez Belhaj ${ }^{\mathrm{a}}$, Alain Vergnet ${ }^{\mathrm{b}}$, \\ Hugues de Verdal $^{\text {c,d }}$, Tristan Rouyer ${ }^{\mathrm{b}}$, Sylvain Bonhommeau ${ }^{\mathrm{e}}$, Achraf Lamlih ${ }^{\mathrm{a}}$, Mohan Julien ${ }^{\mathrm{a}}$, \\ Fathi Ben Ali ${ }^{a}$, Michel Renovell ${ }^{a}$, Serge Bernard ${ }^{a}$, Fabien Soulier ${ }^{\mathrm{a}}$ \\ ${ }^{\text {a } L I R M M, ~ U n i v ~ M o n t p e l l i e r, ~ C N R S, ~ M o n t p e l l i e r, ~ F r a n c e ~}$ \\ ${ }^{\mathrm{b}}$ IFREMER, Univ Montpellier, MARBEC, Sète, France \\ ${ }^{\mathrm{c}}$ CIRAD, UMR ISEM, Montpellier, France \\ ${ }^{\mathrm{d}}$ ISEM, Univ Montpellier, CNRS, EPHE, IRD, Montpellier, France \\ ${ }^{\mathrm{e}}$ IFREMER/DOI, Le Port, France
}

A R T I C L E I N F O

\section{Keywords:}

Bioimpedance

Spectroscopy

Dicentrarchus labrax

Oocyte developmental stages

\begin{abstract}
A B S T R A C T
Fish oocyte development monitoring is a mandatory operation when studying breeding in captivity as well as in the wild. In aquaculture, it is required to determine the best fertilization time. In the wild, it helps to detect spawning grounds, the distribution of fish species and the interaction between animals and their environment. In both settings, the conventional technique for developmental stage identification consists of handling the fish for oocyte sampling and sample observation using a binocular zoom head. Such an operation is difficult for the operator as well as for the fish. There is a need for repeated anesthesia and oocyte sampling, and it relies on operator expertise to identify the developmental stage. In this context, this publication proposes, for the first time, to study the potential of bioimpedance measurement as an alternative in fish breeding studies. We have set up an experiment combining the in vitro bioimpedance measurement of sampled oocytes with the conventional estimation technique for 69 sampled collected on farmed European sea bass. The statistical analysis has demonstrated that three of the four main developmental stages can be identified using bioimpedance measurement. The integrability of the bioimpedance technique with its implantable sensor makes this potential alternative approach very promising. Thanks to this sensor it could be possible, for the first time ever, to monitor in vivo the oocyte developmental stage in captivity as well as in the wild.
\end{abstract}

\section{Introduction}

\subsection{Context}

Animal breeding has been a long-standing research topic, one that has been explored extensively because of the domestication of farmed animals (Lush, 1943). The number of scientific contributions has substantially increased with industrial farming. Nowadays, two fields are clearly distinguishable - breeding in captivity and breeding in the wild.

Studies of fish breeding in both captivity and the wild require an understanding and tracking of the oocyte cycle. Monitoring the oocyte cycle is a key step in artificial reproduction because it allows the detection of the best fertilization times and thus improves the result of artificial insemination of farmed animals. Regarding breeding in the wild, tracking oocyte cycles helps the detection of spawning grounds, the distribution of fish species, and the interaction between animals and their environment (Roth and Kutschera, 2008).

In aquaculture, the conventional technique consists of observing an oocyte sample for developmental stage identification. To do so, females are anesthetized and samples are taken from ovaries using a Pipelle before being observed using a binocular zoom head (Harvey and Hoar, 1980). The observation of the most advanced stage of a sample's oocyte development is used to determine the timing for egg laying. This method is time-consuming and constraining for operators. It requires repeated anesthesia, oocyte sampling and oocyte developmental stage identification for a large number of fish. In addition, it relies heavily on the expertise of the operator in charge of oocyte developmental stage identification. If the method is not appropriately applied, it can lead to

\footnotetext{
* Corresponding author.

E-mail address: vincent.kerzerho@lirmm.fr (V. Kerzérho).
} 
oocyte atresia and disrupt the reproductive cycle of the females (Chen, 1977). It is important to note that although the oocyte developmental process is well known, determining the average developmental stage of ovaries is difficult because of the heterogeneity of oocyte development in them. In order to support the artificial reproduction of fish and to reduce constraints on both operators and fish, an alternative method for oocyte developmental stage monitoring would be very useful. This study investigates whether bioimpedance could provide that solution.

Electrical impedance is a parameter providing a measurement of the opposition of a circuit to an electrical current. By extension, impedance can be measured on any conductive material and, in particular, on biological tissues - which is called bioimpedance. The bioimpedance measurement refers to the electrical property of a biological tissue. For measuring bioimpedance, an alternating current signal is sent through the tissue, then the induced voltage signal is measured (Lamlih, 2018). The intracellular (ICF) and extracellular (ECF) (Fig. 1) fluids contain ions. Due to these free ions (mostly $\mathrm{Na}^{+}$and $\mathrm{K}^{+}$), ECF and ICF are considered as electrolytes, which means that they have the ability to conduct electric current in the presence of an external electrical field. Therefore, the biological tissue can be considered as an ionic conductor (Jaffrin and Morel, 2008; Schlebusch et al., 2010). The cell membrane is predominately constituted of polar lipids. The bilayer lipid membranes (BLM) are responsible for the capacitive nature of cells and by extension the biological tissue. It is interesting to observe the frequency dependency introduced by the cell membrane capacitance. At low frequencies $(<100 \mathrm{kHz})$ the cell membrane acts as an insulator blocking the current from penetrating into the intracellular space (Liedtke, 1997; Ivorra, 2002). However, at high frequencies $(>100 \mathrm{kHz})$ the impedance of the cell membrane decreases, and the current flows through the extracellular space as well as the intracellular space (Liedtke, 1997; Ivorra, 2002). The biological tissue is dispersive because its permittivity and conductivity are functions of frequency. This frequency dependency has been identified by Schwan (Schwan, 1957).

Bioimpedance was first developed for medical purposes, before being used in many veterinary applications. In recent decades, bioimpedance measurements have been used to monitor the development of the estrous cycle in domesticated mammalian species, particularly rats and cows. According to the results of these studies, the increase in impedance correlates with the proestrus stage (Bartos, 1977; Bartos and Sedlacek, 1977; Taradach, 1982; Ramos et al., 2001; Ahmed et al., 2018; Jaramillo et al., 2012). It is important to note that all studies were conducted at a frequency of $1 \mathrm{kHz}$ using a vaginal probe for the bioimpedance measurements. Bioimpedance use seems to be spreading because it has many advantages. It is faster and less restrictive than smear tests, which is the conventional method for oocyte development monitoring (Bartos, 1977; Bartos and Sedlacek, 1977; Taradach, 1982; Ramos et al., 2001; Ahmed et al., 2018). Moreover, there is evidence that bioimpedance disrupts the oocyte cycle less than the conventional method (Taradach, 1982) because it is less invasive and does not require the repeated anesthesia of the female. Finally, this method avoids the possibility that a poorly executed manual sampling could cause oocyte atresia (Chen, 1977).

Bioimpedance is also used in other applications related to fish studies. For instance, bioimpedance measurements have been used to optimize nutritional requirements through the estimation of body composition (Duncan et al., 2007; Andrade et al., 2014). It is considered as an effective tool for assessing the body compositions of different animals in a fast, non-lethal and low-cost manner (Zaniboni-Filho et al., 2015). In addition, the analysis of body composition is also important in wild populations analysis as it is an indicator of their potential responses to factors such as competition, habitat degradation and climate change (Hartman et al., 2011; Willis and Hobday, 2008). To our knowledge there is no existing published study dealing with bioimpedance measurements performed on fish oocytes.

European sea bass (Dicentrarchus labrax) was chosen as a model, because it is one of the main commercially farmed species in the Mediterranean area (Bagni, 2005) and so its oocyte cycle is well known (Mayer et al., 1990). The developmental cycle consists of seven stages (Fig. 2). In October, the oocytes enter into vitellogenesis. At this time, the egg yolk precursor proteins (vitellogenin) formed in the liver are driven out and stored in the oocytes (Sullivan and Yilmaz, 2018). Oocytes in vitellogenesis are recognizable by their small diameter (less than $800 \mu \mathrm{m})$ as well as their opacity after an Ethanol, Formalin and Acetic acid (EFA) addition of a volume equal to the volume of the oocytes sample. When reaching a diameter of $800 \mu \mathrm{m}$, they enter the post-vitellogenic stages. There are four post-vitellogenic stages: A, B, C and D. It is possible to differentiate them by the position of the lipid droplets and the nucleus in the cell (Fig. 2). The cells then fill with water, which makes them translucent and increases their diameter to more than $1 \mathrm{~mm}$ - this is the hydrated stage. If the oocytes are neither hydrated nor expelled from the ovary to be fertilized, they enter into atresia and regress (Polder, 1962). At this point, the membrane is absorbed and the cell shrinks until it disappears. These cells are opaque and have a rough appearance. The breeding period of the sea bass concludes at the end of March and females can lay eggs up to twice a year. As a consequence, the developmental stages of oocytes in the gonad may be heterogeneous. Because of this low number of egg-layings per year, it is important to be able to precisely predict their development cycle. If the egg-laying moment is missed, the oocytes will be in atresia and reproduction missed.

This experiment focuses on the use of bioimpedance in the context of fish breeding, through the manual sampling of oocytes to combine in vitro bioimpedance measurements with the observation of their developmental stages. The main objective was to examine whether a relationship between the two could be found. Bioimpedance varies with measurement frequency but the current body of fish studies using
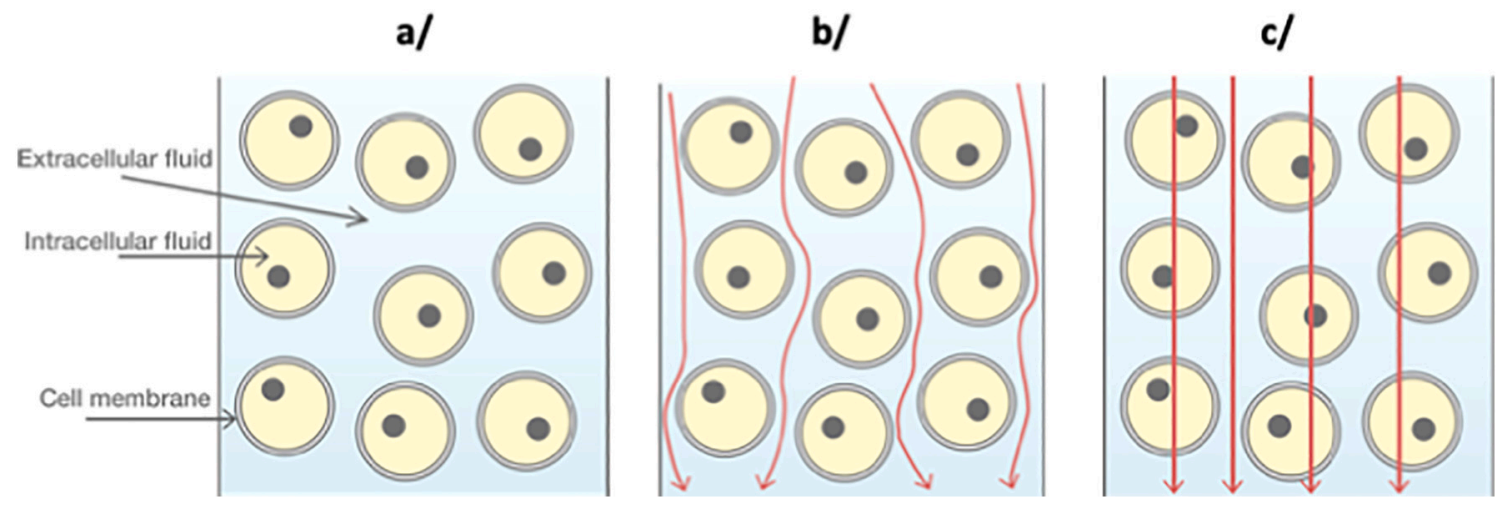

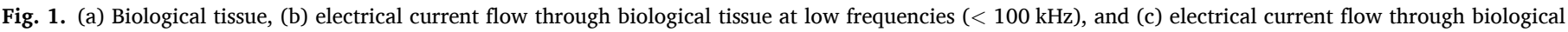
tissue at high frequencies $(>100 \mathrm{kHz})$. 


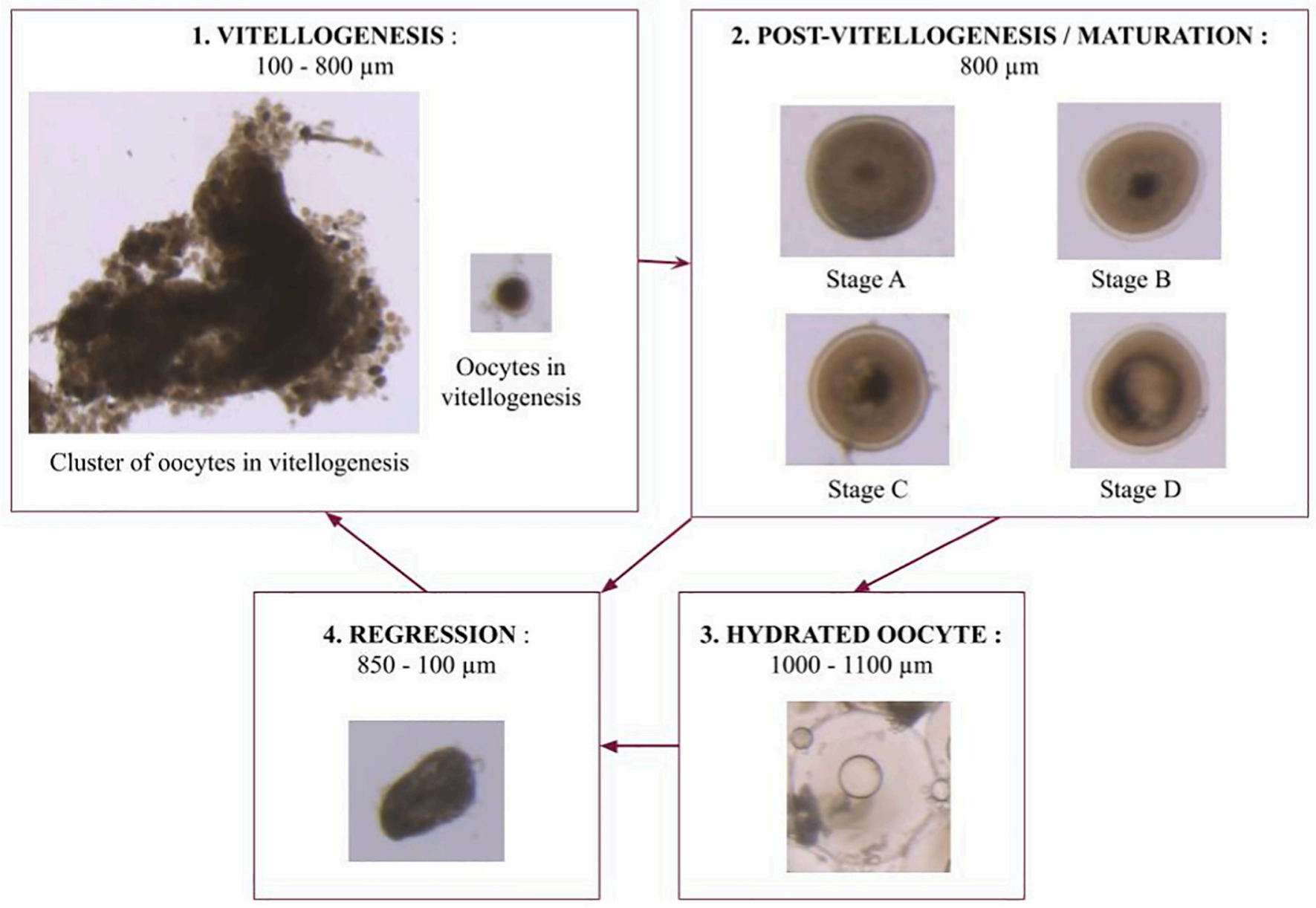

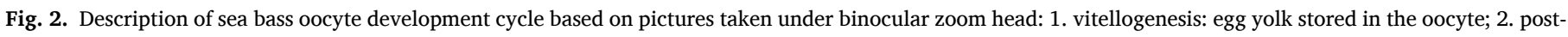

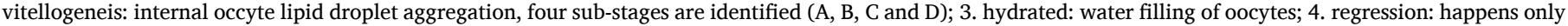
if the oocytes are not expelled.

bioimpedance analysis has only used single-frequency measurements, not allowing for the investigation of the effect of varying frequency. This will be tested in this study through bioimpedance spectroscopy for the particular case of oocyte-cycle monitoring.

\section{Materials and methods}

\subsection{Biological sampling and visual determination of developmental stages}

The experiment took place in the IFREMER experimental platform at Palavas-les-Flots, France.

For the experiment, 69 samples of oocytes have been collected during one season, between 3rd to 28th of March 2019. The samples have been collected on 22 sea bass females. Six females were homozygous albinos, with an average weight of $5.9 \mathrm{~kg}$ and an average age of 9 years old. The other 16 females have been selected over three generations for their growth rate. Their average weight was $3.4 \mathrm{~kg}$ and their average age was 7 years old. The fishes were firstly sedated adding $15 \mathrm{~g}$ per $\mathrm{m}^{3}$ of Benzocaine in the rearing tank to be fished easily. In a second time, the fish selected for sampling was put in a tank with a higher dose of Benzocaine ( $45 \mathrm{~g} \mathrm{per}^{3}$ ) with oxygen supply to be anesthetized. The loss of body movements but with continued opercular movements was obtained after $5 \mathrm{~min}$. The $1 \mathrm{~mL}$ Pipelles used for oocyte sampling were completely filled so that the volume analyzed remained constant. There was not any euthanasia. The recovery of the equilibrium comes $5 \mathrm{~min}$ after the return of the fish in the sea water. All procedures were in accordance with the French and the EU legislation regarding animal experimentation (APAFIS, Permission No.2020022814315445-24425)

To assign a developmental stage to the sample, one drop of the sample was placed in a petri dish. Then to obtain a mix with equivalent volumes of the two elements, $1 \mathrm{~mL}$ of a solution composed of Ethanol, Formalin and Acetic acid (EFA) was added to fixate the sample and light up the oocytes. After $10 \mathrm{~min}$, under the effect of EFA, oocytes dissociate and their cytoplasm discolors, which allows for the determination of oocyte developmental stages under a binocular microscope (Parfouru and Fauvel, 1998). Samples composed of hydrated oocytes did not require EFA to be determined, which also darkens the cells at that developmental stage. Pictures of the discolored oocytes were taken under a binocular zoom head, which was connected to a camera and used with image acquisition software (LAS 4.9). Five photos were taken for each sample. As previously mentioned, the development of oocytes in a gonad is not uniform. As a consequence, to determine the development stage of a gonad, the majority of developmental stages for each sample were determined by visual analysis. When there was a second stage in large quantity in the sample, it has been noted as a secondary development stage. This results in samples with two development stages such as regression and vitellogenesis. 


\subsection{Bioimpedance measurements}

Bioimpedance is a complex number, whose cartesian form is given by Eq. (1).

$Z=\mathrm{R}+\mathrm{iX}$

Where $Z$ is impedance, $R$ is the real part - also called resistance - and $X$ is the imaginary part - also called reactance. The measurement principle (Fig. 3) consists of generating a current signal (I), measuring the induced voltage $(U)$ and computing the ratio between voltage and current $-Z=$ $\frac{U}{I}$.

The current signal is a sine wave, and one of its characteristics is its frequency. Bioimpedance spectroscopy consists of measuring bioimpedance at different current frequencies. In this experiment, bioimpedance spectroscopy was measured over a large band, from $80 \mathrm{~Hz}$ to $5 \mathrm{MHz}$, using a MFIA bioelectrical impedance analyzer from Zurich Instruments (Fig. 4b). The oocytes were sampled (Fig. 4a) to observe their developmental stages and the bioimpedance measurements were done in vitro (Fig. 4d) using an electrode inside a well (Fig. 4c) connected to the impedance analyzer (Fig. 4b). The orange strip electrode was partly inserted in the well, which was 3D printed in Polylactic Acid (PLA) (Fig. 4c). The part of the electrode inside the well held the two metallic contacts used as an interface between the electronics and oocyte for bioimpedance measurement. The part of the electrode outside was connected to the impedance analyzer through a connector and two wires. Details on the electrode architecture are provided as supplementary information (SI1 fig.8). The volume of the well was $1.77 \mathrm{~cm}^{3}$, which is slightly bigger than the volume of the Pipelle usually used to sample sea bass oocytes.

The laboratory was set at the constant temperature of $17^{\circ} \mathrm{C}$ to avoid any potential effects of temperature on the bioimpedance measurement (Hartman et al., 2011).

\subsection{Analysis}

A, B, C and D stages were grouped together and called postvitellogenesis in order to increase the number of samples per stage. We then considered the samples within one of these three stages: vitellogenesis (vit), post-vitellogenesis (post-vit) and hydrated (hyd). The samples in the regression stage were removed from the results because it is a stage where oocytes progressively shrink and split for fat gathering, resulting in a wide variety of biological states. This variety would result in a large range of bioimpedances, which potentially hides information related to other developmental stages. In addition, six

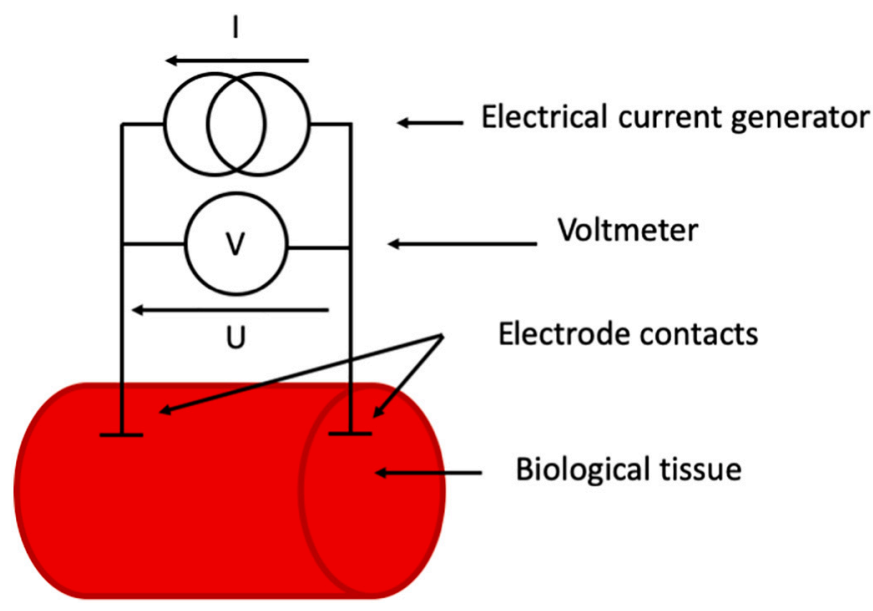

Fig. 3. Bioimpedance measurement principle - an electrode is used to interface the electronics with the biological tissue, an alternating current (I) is generated and the induced voltage (U) is measured using a voltmeter. outliers were removed because they had a high heterogeneity of stage or they contained blood. Blood is a very good conductor, and can skew measurements. A principal component analysis (PCA) was done to study the relationship between the developmental stages and bioimpedance modulus. We used the bioimpedance modulus at each of the 201 frequencies as variables, and the PCAs were performed using the ade $4 \mathrm{R}$ package, and were centered and normalized. The projections of individuals onto the first and second principal axes, which explains the most variance, were used to visualize whether groups emerged and also their relationship to the bioimpedance frequency range.

\section{Results}

\subsection{Principal component analysis}

According to Fig. 6 we can clearly distinguish three clusters. They group three different oocyte developmental stages, which are the vitellogenesis, post-vitellogenesis and hydrated stages.

According to the placement of elipses in Fig. 6 and the placement of variables on the circle (Fig. 5), we can say that the modulus of samples measured at the vitellogenesis stage were higher at low frequencies (below $200 \mathrm{kHz}$ ). Conversely, the modulus measured for the samples in the post-vitellogenesis developmental stage were higher at high frequencies. Finally, the hydrated samples were found to be independent from the frequency at which the measure was done and the modulus of measures were low regardless of the frequency.

\subsection{Bioimpedance spectra comparison}

As the PCA results shed light on some bioimpedance modulus amplitude differences between the vitellogenesis, post-vitellogenesis and hydrated stages, we plotted the related spectra (Fig. 7) and noticed spectra modulus at few frequencies (Table 1.)

Based on the comparison of spectra values, we can provide similar conclusions as the ones for PCA analysis. Considering a frequency, we can distinguish the three development stages. The modulus was the lowest regardless of the frequency for hydrated samples. At low frequencies (below $200 \mathrm{kHz}$ ), the modulus was highest for vitellogenesis samples and the modulus was twice as high for the vitellogenesis stage as for the hydrated stage. Finally, at higher frequencies (above $200 \mathrm{kHz}$ ), the modulus was highest for post-vitellogenic samples.

\section{Discussion}

For the first time, we are studying the potential correlation between the bioimpedance of fish oocyte and their developmental stages. In addition, we are providing an unprecedented interpretation of bioimpedance spectrum modulus variations over the oocyte developmental cycle. For the purpose of this study, we set up an experiment consisting of sampling oocytes of farmed sea bass at different developmental stages, measuring bioimpedance using an in vitro impedance measurement system and estimating their developmental stage using a conventional approach. For analysis purposes, instead of measuring bioimpedance at a single frequency, a wide band bioimpedance spectroscopy was measured. The relationship analysis has been done using statistical analysis and spectra comparison. Thanks to this experiment, we have demonstrated that by using bioimpedance spectroscopy it is possible to differentiate three of the four main developmental stages.

The bioimpedance modulus measured at the hydrated stage was found to be lowest at all frequencies, meaning that the current flowed more easily through the samples regardless of the frequency. At this developmental stage, the accumulation of ions and the increased quantity of free amino acids created by the cleavage of the lipid droplets generate the osmotic mechanism, during which the water in the extracellular medium enters the cells (Sullivan and Yilmaz, 2018). Aquaporins-1o participates in the processes by acting as channels 

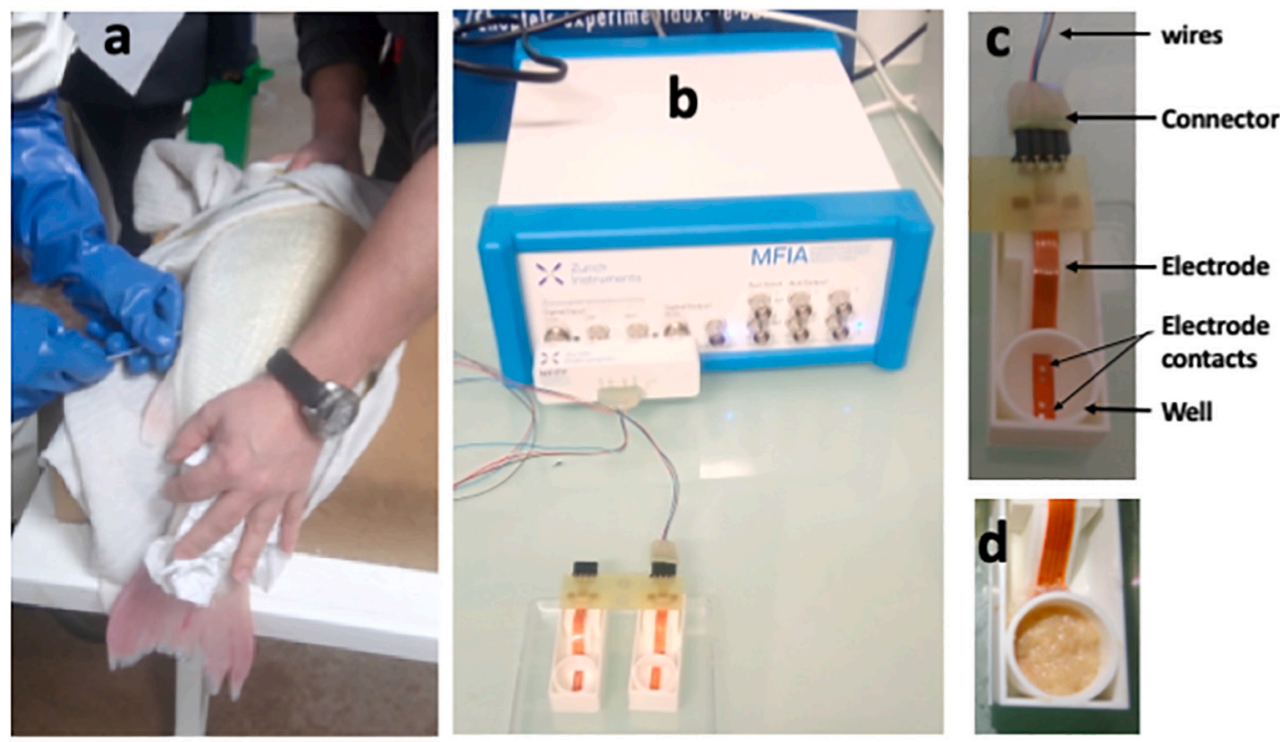

Fig. 4. (a) oocyte sampling from a female sea bass using a Pipelle, (b) bioimpedance measurement set-up of impedance analyzer connected to a well with an electrode, (c) well with an electrode partially inserted - the part in the well holds the contacts used as an electrical interface with the oocytes, the part outside is connected to wires from the impedance analyzer, (d) well filled with sampled oocytes. (For interpretation of the references to color in the text, the reader is referred to the web version of this article.)

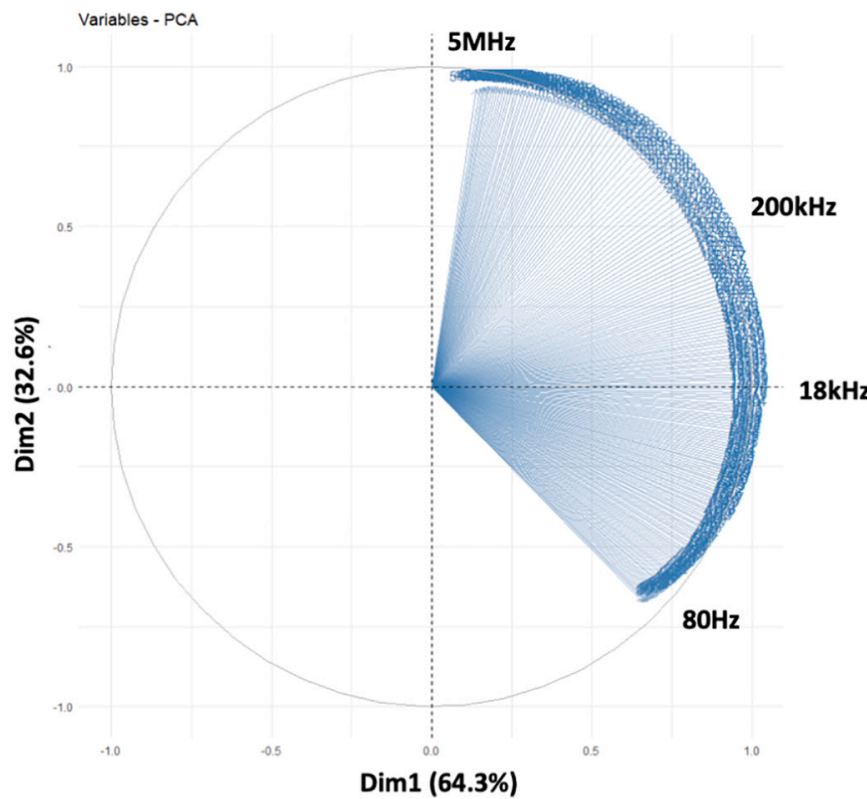

Fig. 5. Two-dimensional representation of the contribution of 201 variables (modulus at 201 frequencies, from $80 \mathrm{~Hz}$ to $5 \mathrm{MHz}$ ) to the inertia. The variables are placed in ascending order of frequency. The 201 variables are strongly correlated, reflecting the small variations of modulus between two successive frequencies. The 1st (64.3\%) and the 2nd (32.6\%) axes of the PCA explain more than $96 \%$ of the total variance.

between the extra and intracellular medium. Thus, aquaporins could become gateways to the stream for easier access to the intracellular medium. In addition, once inside the cell, the current flows easily through the presence of more water than for the other stages.

Published studies on the ovarian cycle of rats (Rattus norvegicus) described an increase of bioimpedance modulus during the proestrus phase at $1 \mathrm{kHz}$ (Bartos, 1977; Taradach, 1982; Ramos et al., 2001;

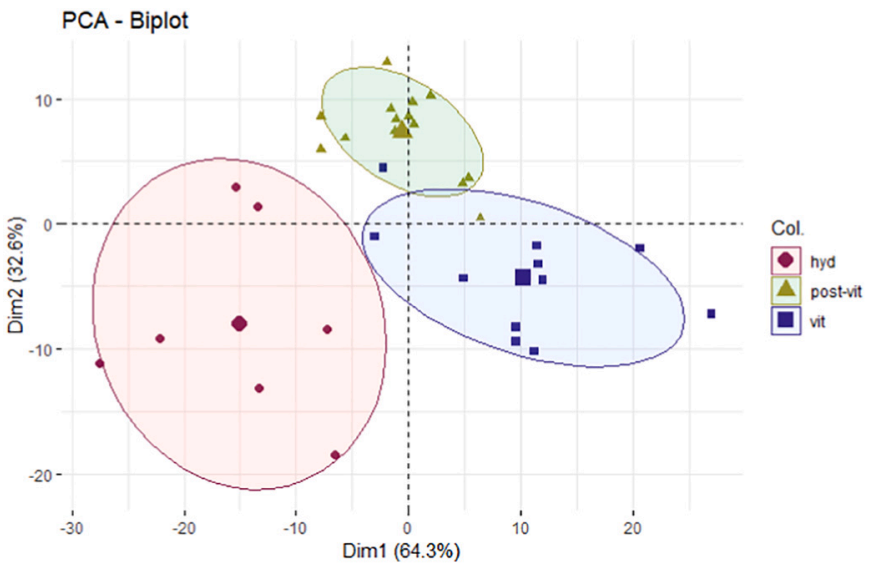

Fig. 6. 2D reduced dimension dataset using the PCA without outliers, without regression stage, with elipses.

Jaramillo et al., 2012). For other species, especially cows and guinea pigs, studies have shown similar results (Ahmed et al., 2018; Bartos and Sedlacek, 1977). Our results are in line with this literature, as the bioimpedance modulus of samples from the vitellogenesis developmental stage were found higher at low frequencies. Indeed the bioimpedance modulus of the samples in the vitellogenesisis stage were found to be the highest below $200 \mathrm{kHz}$ (Figs. 6 and 7). At low frequencies $(<100 \mathrm{kHz})$, the cell membrane acts as an insulator avoiding current penetration into the intracellular space(Liedtke, 1997; Ivorra, 2002). When oocytes are in vitellogenesis, a tissue connects oocytes to each other and a vascular system composed of red blood cells is developed. Both allow the oocytes to be fed with neutral lipids (Sullivan and Yilmaz, 2018). Lipids are therefore present in the extracellular space. According to literature (Jun et al., 2012; Legin et al., 2007), at frequencies lower than $100 \mathrm{kHz}$, the increase of fat concentration in biological tissues induces an increase of the bioimpedance modulus. That could explain why the vitellogenesis stage is the one with the highest bioimpedance modulus.

The stage of regression has the "widest" disparity of bioimpedance 


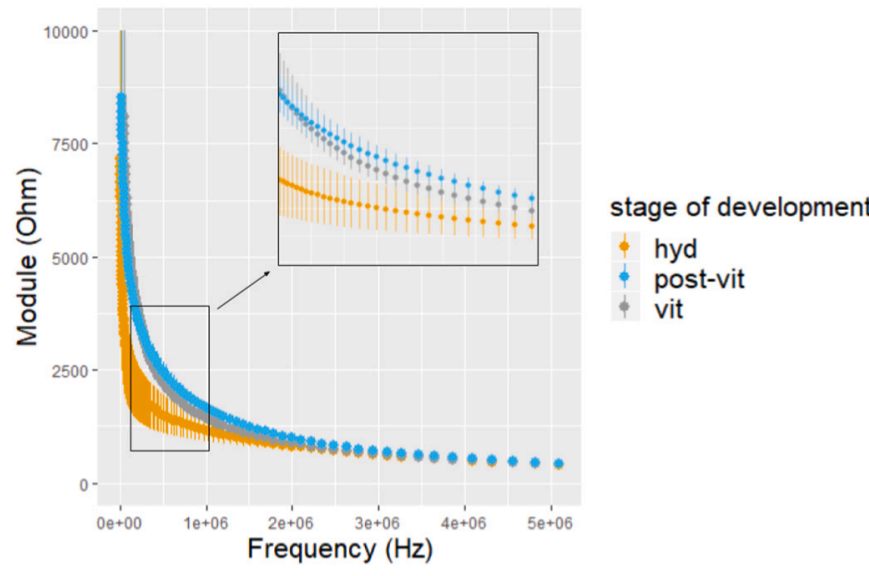

Fig. 7. Average/max/min of spectra for three developmental stages: hydrated (hyd), post-vitellogenesis (post-vit) and vitellogenesis (vit).

Table 1

Measured moduli averages $(\mathrm{Ohm})$ for each stage at different frequencies.

\begin{tabular}{llll}
\hline Frequency & Vitellogenesis & Post-vitellogenesis & Hydrated \\
\hline $1 \mathrm{kHz}$ & $36,388.51$ & $25,300.04$ & $21,942.35$ \\
$10 \mathrm{kHz}$ & $12,961.44$ & 9861.11 & 6322.68 \\
$100 \mathrm{kHz}$ & 5386.60 & 4787.64 & 2635.21 \\
$200 \mathrm{kHz}$ & 3403.09 & 3445.74 & 2041.75 \\
$1 \mathrm{MHz}$ & 1354.04 & 1558.90 & 1145.41 \\
$3 \mathrm{MHz}$ & 657.83 & 720.30 & 630.22 \\
\hline
\end{tabular}

modulus avoiding a clear bioimpedance signature for this stage. This may be due to the fact that this stage is the most heterogeneous from a biological point of view. First, the size of the cells fluctuates very significantly between the beginning of the regression (where the cells are $1 \mathrm{~mm}$ in diameter) and the total disappearance of the cells - as the cells degenerate and are resorbed by the ovarian stroma (Leonardo et al., 2006; Asturiano et al., 2000). The follicular wall, which was previously present around the cell, folds before disappearing. In addition, the lipid droplets and water previously stored in the cells are released into the extracellular medium. During the entire process of degradation, the ease of the current to flow can be disturbed and can vary significantly from one sample to another. This may explain the important disparity of this stage. Finally, other stages may be present at the same time. The heterogeneity of the samples can therefore bias the observed results. The regressions stage is the last stage of the oocyte cycle. In relation to artificial reproduction, this is the least relevant stage. If it does happen, it means there is no possible mating for the female. For artificial reproduction, females receive a hormonal stimulation in an earlier stage in order to make them release their oocytes.

Post-vitellogenesis samples have the highest impedance at high frequencies ( $>200 \mathrm{kHz}$ ) (Liedtke, 1997; Ivorra, 2002). This may be explained by the fact that the oocytes are filled with lipid droplets and have a more elevated density of lipids in the intracellular medium than the other stages.

With in vitro measurement case studies, it is of interest to look for additional statistical analysis to get a more precise identification of developmental stages. We are currently considering an additional experiment that would use supervised machine-learning to train a correlation model between bioimpedance spectroscopy and oocyte developmental stages. Thanks to such a model we could expect to identify the developmental stages of new oocyte samples using bioimpedance.

The next development regarding the use of bioimpedance as a measurement technique for oocyte developmental stage identification will be to take the measurements inside the gonad. With this approach, we would avoid oocyte sampling.
Bioimpedance spectroscopy is a non-destructive measurement technique. Another advantage of such a technique is its potential integrability. It can even be deployed using limited electrical resources (Lamlih, 2018). As a result, a very promising application of bioimpedance measurement would be the in vivo implantation of a sensor. Such a device could provide accurate monitoring of oocyte development with fish handling limited only to the sensor implantation. This kind of approach could provide additional knowledge on fish oocyte cycle in captivity as much as in the wild, providing a completely new and groundbreaking perspective on fish studies.

\section{Declaration of Competing Interest}

The authors declare that they have no known competing financial interests or personal relationships that could have appeared to influence the work reported in this paper.

\section{Acknowledgments}

This work was supported by the French National Research Agency under the Investments for the Future Program, referred as ANR-16CONV-0004.

\section{Appendix A. Supplementary data}

Supplementary data to this article can be found online at https://doi. org/10.1016/j.aquaculture.2021.737396.

\section{References}

Ahmed, M., Chowdhury, M., Rahman, M., Bhattacharjee, J., Bhuiyan, M., 2018. Relationship of electrical resistance of vaginal mucus during oestrus with post-ai pregnancy in cows. Bangladesh J. Vet. Med. 15, 113. https://doi.org/10.3329/bjvm. v15i2.35520.

Andrade, F., Abreu, M., Lopes, J., Valente de Figueiredo, A., Araripe, M., Hosmylton Carvalho Ferreira, A., 2014. Ichthyometry and electrical bioimpedance analysis to estimate the body composition of tambatinga. Acta Amaz. 44, 279-285. https://doi. org/10.1590/S0044-59672014000200014.

Asturiano, J., Sorbera, L., Ramos, J., Kime, D., Carrillo, M., Zanuy, S., 2000. Hormonal regulation of the European sea bass reproductive cycle: an individualized female approach. J. Fish Biol. 56, 1155-1172. https://doi.org/10.1006/jfbi.2000.123.

Bagni, M., 2005. Report Cultured Aquatic Species Information Programme, Dicentrarchus labrax. Food and Agriculture Organization of the United Nations Fisheries and Aquaculture Department [online]. Technical Report.

Bartos, L., 1977. Vaginal impedance measurement used for mating in the rat. Lab. Anim. 11, 53-55. https://doi.org/10.1258/002367777780959148.

Bartos, L., Sedlacek, J., 1977. Vaginal impedance measurement used for mating in the guinea-pig. Lab. Anim. 11, 57-58. https://doi.org/10.1258/002367777780959210.

Chen, T., 1977. Aquaculture practices in Taiwan, Farnham, surrey: fishing news (books). J. Marine Biol. Assoc. U. K. 57 https://doi.org/10.1017/S0025315400021433, 283-283.

Duncan, M., Craig, S., Lunger, A., Kuhn, D., Salze, G., McLean, E., 2007. Bioimpedance assessment of body composition in cobia Rachycentron canadum (1. 1766). Aquaculture 271, 432-438. https://doi.org/10.1016/j.aquaculture.2007.06.002.

Hartman, K.J., Phelan, B.A., Rosendale, J.E., 2011. Temperature effects on bioelectrical impedance analysis (bia) used to estimate dry weight as a condition proxy in coastal bluefish. Marine Coast. Fish. 3, 307-316. https://doi.org/10.1080/ 19425120.2011 .603961$.

Harvey, B., Hoar, W., 1980. Reproduction Provoquée chez les Poissons: théorie et Pratique, pp. 1-51.

Ivorra, A., 2002. Bioimpedance Monitoring for Physicians: An Overview. Centre Nacional de Microelectrònica Biomedical Applications Group 1.

Jaffrin, M., Morel, H., 2008. Body fluid volumes measurements by impedance: a review of bioimpedance spectroscopy (bis) and bioimpedance analysis (bia) methods. Med. Eng. Phys. 30, 1257-1269. https://doi.org/10.1016/j.medengphy.2008.06.009.

Jaramillo, L., Balcazar, I., Duran, C., 2012. Using vaginal wall impedance to determine estrous cycle phase in lewis rats. Lab. Anim. 41, 122-128. https://doi.org/10.1038/ laban0512-122.

Jun, H.S., Dao, L.T.M., Cho, S., 2012. Electrical impedance detection of senescence in adipose tissue-derived stem cells. Proc. Eng. 47, 1025-1028. https://doi.org/ 10.1016/j.proeng.2012.09.323. http://www.sciencedirect.com/science/article/pii/ S187770581204386X. , (26th European Conference on Solid-State Transducers, EUROSENSOR 2012)

Lamlih, A., 2018. Design of an Integrated Bioimpedance Measurement System for Chronic Monitoring of Biological Tissue Composition. Ph. D. Thesis. University of Montpellier. http://www.theses.fr/2018MONTS070. 
Legin, M., Laputková, G., Sabo, J., Vojcíková, L., 2007. Impedance spectroscopy of bilayer lipid membranes self-assembled on agar support - interaction with hdl. Physiol. Res. Acad. Sci. Bohemoslov. 56 (Suppl 1), S85-S91.

Leonardo, A., Romagosa, E., Batlouni, S., Borella, M., 2006. Occurrence and significance of ovarian and follicular regression in cachara Pseudoplatystoma fasciatum (linnaeus, 1766): a histology approach. Arquivo Brasileiro De Medicina Veterinaria E Zootecnia - Arq Bras Med Vet Zootec 58. https://doi.org/10.1590/S0102 09352006000500019.

Liedtke, R., 1997. Principles of Bioelectrical Impedance Analysis. RJL Systems Inc. Clinton, MI 23, pp. 1226-1243. https://doi.org/10.1016/j.clnu.2004.06.004.

Lush, J., 1943. Animal Breeding Plans. The Iowa State College Press, p. 457.

Mayer, I., Shackley, S., Witthames, P., 1990. Aspects of the reproductive biology of the bass, Dicentrarchus labrax $L$. ii. fecundity and pattern of oocyte development. J. Fish Biol. 36, 141-148. https://doi.org/10.1111/j.1095-8649.1990.tb05590.x.

Parfouru, D., Fauvel, C., 1998. Développement ovocytaire chez l'ombrine, Sciaenops Ocellatus, en conditions tropicales martiniquaises. In: Proceedings of the 50th Gulf and Caribbean Fisheries Institute, pp. 551-573.

Polder, J., 1962. Cyclical changes in testis and ovary related to maturity stages in the north sea herring, Clupea Harengus L. Arch. Néerlandaises de Zool. 14, 45-60. https://doi.org/10.1163/036551661X00025.

Ramos, S., Lee, J., Peuler, J., 2001. An inexpensive meter to measure differences in electrical resistance in the rat vagina during the ovarian cycle. J. Appl. Physiol. 91, 667-670. https://doi.org/10.1152/jappl.2001.91.2.667.

Roth, T., Kutschera, U., 2008. Darwin's hypotheses on the origin of domestic animals and the history of German shepherd dogs. Ann. Hist. Philos. Biol. 13, 175-187.
Schlebusch, T., R\&ldquo;othlingsh\&rdquo;ofer, L., Kim, S., Köny, M., Leonhardt, S., 2010. On the Road to a Textile Integrated Bioimpedance Early Warning System for Lung Edema, pp. 302-307. https://doi.org/10.1109/BSN.2010.21.

Schwan, H.P., 1957. Electrical properties of tissue and cell suspensions* *this work was supported in part by grants from the United States public health service, h-1253(c24) and in part by the office of naval research, 119-289. Volume 5 of Advances in Biological and Medical Physics. Elsevier, pp. 147-209. https://doi.org/10.1016/ B978-1-4832-3111-2.50008-0. http://www.sciencedirect.com/science/article/pii B9781483231112500080.

Sullivan, C.V., Yilmaz, O., 2018. Vitellogenesis and Yolk Proteins, Fish, pp. 266-277. https://doi.org/10.1016/B978-0-12-809633-8.20567-0. https://www.sciencedirect. com/science/article/pii/B9780128096338205670.

Taradach, C., 1982. Monitoring of the oestrus cycle in the rat by measurement of vaginal impedance. In: Chambers, C.M., Chambers, P.L. (Eds.), New Toxicology for Old. Springer Berlin Heidelberg, Berlin, Heidelberg, pp. 184-186.

Willis, J., Hobday, A.J., 2008. Application of bioelectrical impedance analysis as a method for estimating composition and metabolic condition of southern bluefin tuna (thunnus maccoyii) during conventional tagging. Fish. Res. 93, 64-71. https://doi. org/10.1016/j.fishres.2008.02.010. http://www.sciencedirect.com/science/article/ pii/S0165783608000659.

Zaniboni-Filho, E., Hermes-Silva, S., Weingartner, M., Jimenez, J., Borba, M., Fracalossi, D., 2015. Bioimpedance as a tool for evaluating the body composition of suruvi (Steindachneridion scriptum). Braz. J. Biol. 75, 239-244. https://doi.org/ 10.1590/1519-6984.13614. 